\title{
PERSPECTIVE OPEN Broken symmetries, non-reciprocity, and multiferroicity
}

\author{
Sang-Wook Cheong ${ }^{1}$, Diyar Talbayev ${ }^{2}$, Valery Kiryukhin ${ }^{1}$ and Avadh Saxena ${ }^{3}$
}

The interplay of space and time symmetries, ferroic properties, chirality and notions of reciprocity determines many of the technologically important properties of materials such as optical diode effect, e.g., in polar ferromagnet $\mathrm{FeZnMo}_{3} \mathrm{O}_{8}$. We illustrate these concepts, including the non-reciprocal directional dichroism, through a number of practical examples. In particular, the conditions for non-reciprocity of ferro-rotational order are discussed and the possible use of linear optical gyration is suggested as a way to detect ferro-rotational domains. In addition, we provide the means to achieve high-temperature optical diode effect and elucidate multiferroic behaviors as a result of helical vs. cycloidal spins. Finally, we identify different entities behaving similarly under all symmetry operations, which are useful to understand non-reciprocity and multiferroicity in various materials intuitively.

npj Quantum Materials (2018)3:19; doi:10.1038/s41535-018-0092-5

When the motion of an object in one direction is different from that in the opposite direction, it is called a non-reciprocal effect. ${ }^{1}$ The object can be an electron, a phonon (lattice wave), a magnon (spin wave), or light in crystalline solids, and the best known example is that of non-reciprocal charge transport (i.e., diode) effects in $\mathrm{p}-\mathrm{n}$ junctions, where a built-in electric field $(\boldsymbol{E})$ breaks the directional symmetry. The polarization $(\boldsymbol{P})$ of ferroelectrics can also act like the built-in electric field, so bulk ferroelectric diode (and photovoltaic) effects can be realized. ${ }^{2}$ Certainly, both $\boldsymbol{E}$ and $\boldsymbol{P}$ are polar vectors, and behave identically under various symmetry considerations. In addition to $\mathrm{p}-\mathrm{n}$ junctions, numerous technological devices such as optical isolators, spin current diodes or metamaterials utilize non-reciprocal effects. In this perspective, we will discuss how non-reciprocal effects can arise from broken symmetries in various crystalline materials, especially multiferroics.

Multiferroics are materials where ferroelectric and magnetic orders coexist, and space inversion and time reversal symmetries are simultaneously broken. ${ }^{3}$ Thus, multiferroics are often good candidates for non-reciprocal effects. Magnetic order naturally breaks time reversal symmetry, and a magnetic lattice, combined with a crystallographic lattice, can break space inversion symmetry, leading to multiferroicity, called magnetism-driven ferroelectricity. In this perspective, two types of magnetism-driven ferroelectricity will also be considered in terms of various symmetries.

Symmetry governs physics, in particular a broken symmetry leads to a phase transition. (ref. 4 and references therein). There are five important symmetries relevant to crystalline materials, namely translational, rotational, mirror reflection, space inversion and time reversal. Note that these symmetries are not completely independent; for example, space inversion operation is equivalent to a $180^{\circ}$ rotation about the vertical axis plus a mirror reflection about the horizontal mirror plane. Full theoretical descriptions and classifications of all crystalline materials and also the requirement for non-reciprocity in crystalline materials in terms of symmetry are well documented in literature, ${ }^{5,6}$ but in this perspective, we will limit our discussion to the central cases most relevant to experiments. Depending on the space inversion and time reversal symmetries, ferroics with a vector order parameter can be divided in four distinct categories as depicted in Fig. 1. The more familiar ferroelectric materials are characterized by electric polarization $(\boldsymbol{P}$, a polar vector) as an order parameter, which is invariant under time reversal but changes sign under space inversion. Similarly, the well-known ferromagnetic materials are described by magnetization ( $\boldsymbol{M}$, an axial vector) as an order parameter, which is invariant under space inversion but changes sign under time reversal. Time reversal is equivalent to changing the direction of the current in the loop in Fig. 1, which is tantamount to reversing the magnetization ref. 7 and references therein. There are two more possibilities, one of which includes materials with an order parameter that changes sign under both the space inversion and time reversal operations. Such materials are termed ferro-toroidal in which magnetization vectors $M$ (or spins) are arranged in a closed loop with radius $r^{8-12}$ The order parameter is called toroidization (or ferro-toroidal moment) $\boldsymbol{T}=\boldsymbol{r} \times \mathbf{M}$. In fact, such an order parameter also relates to magnetoelectricity as we can also write $\boldsymbol{T}=\boldsymbol{P} \times \boldsymbol{M}$ since $\boldsymbol{P}=\mathrm{q} \boldsymbol{r}$ with q being the charge that creates polarization $\boldsymbol{P}$. Toroidization or toroidal moment can arise due to the ordering of magnetic vortices in a material. Note that not all material properties that change sign under time reversal and spatial inversion are ferro-toroidal. An example is $\mathrm{Cr}_{2} \mathrm{O}_{3}$, which is not ferro-toroidal but is described by a pseudo-scalar order parameter, which is time-reversal odd. In this context, the electric dipole transition can also be probed by one-photon experiments such as gyrotropic birefringence and the associated optical magnetoelectric effect, in contrast to second harmonic generation which is a two-photon process. ${ }^{13}$

For the other possibility, we can imagine an ordering of polar vortices described by an order parameter (or ferro-rotation moment) $\boldsymbol{R}=\boldsymbol{r} \times \boldsymbol{P}$ (Fig. 1). ${ }^{10,14}$ However, this order parameter remains invariant under both space inversion and time reversal (although other symmetries such as a mirror reflection can be broken in this case). Such materials are termed ferro-rotational in this perspective and can be realized in, e.g., structural distortions with oxygen cage rotations or nano-structured materials. ${ }^{14}$ This "ferro-rotational" order is sometimes called "electric ferro-toroidal"

\footnotetext{
${ }^{1}$ Rutgers Center for Emergent Materials and Department of Physics and Astronomy, Piscataway, NJ 08854, USA; ${ }^{2}$ Department of Physics and Engineering Physics, Tulane University, 6400 Freret St., New Orleans, LA 70118, USA and ${ }^{3}$ Theoretical Division, Los Alamos National Lab, Los Alamos, NM 87545, USA

Correspondence: Sang-Wook Cheong (sangc@physics.rutgers.edu)
}

Received: 2 January 2018 Revised: 14 February 2018 Accepted: 28 February 2018

Published online: 03 April 2018 


\begin{tabular}{|c|c|c|}
\hline $\begin{array}{l}\begin{array}{c}\text { Space } \\
\text { Inversion }\end{array} \\
\text { Time } \\
\text { Reversal }\end{array}$ & Invariance & Change \\
\hline Invariance & $\begin{array}{c}\text { Ferro-rotational } \\
r \times P\end{array}$ & $\begin{array}{l}\text { Ferro-electric } \\
\qquad P \quad \text { Ferro-chiral }\end{array}$ \\
\hline Change & $\begin{array}{r}\text { Ferro-magnetic } \\
\qquad M\end{array}$ & $\begin{array}{c}\text { Ferro-toroidal } \\
r \times M(P \times M)\end{array}$ \\
\hline
\end{tabular}

Fig. 1 Ferroics with vector order parameters. This table summarizes how vector order parameters change their signs under space inversion and time reversal symmetry operations. Note that space inversion and time reversal symmetries are not broken in ferrorotational order, while both of them are broken in ferro-toroidal order. Ferro-rotational order is sometimes called ferro-axial or electric ferro-toroidal order

or "ferro-axial" order, since $\boldsymbol{r} \times \mathbf{P}$ is an axial vector (i.e., it does not change its sign under space inversion; alternatively, it is a cross product of two polar vectors). The dot product of a polar vector with an axial vector (e.g., E.B) results in a pseudo-scalar. Note that the change of these ferroic order parameters under translation, spatial rotation, and mirror reflection operations is rather straightforward; for example, ferro-rotational moment changes its sign under reflection on the mirror along the moment direction, while ferro-toroidal moment remains invariant under the same operation.

Time derivative of a magnetic field and curl (or rotational derivative) of an electric field $(\mathrm{d} \boldsymbol{B} / \mathrm{d} t \propto \nabla \times \boldsymbol{E})$ as well as magnetic monopole current represent additional examples of ferrorotational order, and can serve as conjugate fields to $\boldsymbol{r} \times \boldsymbol{P}$. Similarly, velocity $(\mathrm{d} \boldsymbol{r} / \mathrm{d} t)$, rotational derivative of a magnetic field $(\nabla \times B)$ and time derivative of an electric field $(\mathrm{d} E / \mathrm{d} t)$ exhibit the symmetries of ferro-toroidal order, and can serve as conjugate fields to $\boldsymbol{r} \times \boldsymbol{M}$ or $\boldsymbol{P} \times \boldsymbol{M}$. To paraphrase, physical quantities with similarly broken symmetries can couple to each other: An electric current, producing $\nabla \times \boldsymbol{B}$, can couple to ferro-toroidal order, and a time-varying magnetic field, producing $\nabla \times \boldsymbol{E}$, can couple to ferrorotational order. Magnetic monopole current (but not spin current), producing $\nabla \times \boldsymbol{E}$, can also couple to ferro-rotational order. Interestingly, any velocity (e.g., that of electrons, phonons, spin waves, light, etc.), changing its sign under either space inversion or time reversal, can in principle couple to ferro-toroidal order. The presence of such coupling implies that the coupled physical quantities can be used to experimentally detect domains associated with a specific ferroic order. ${ }^{15,16}$ In addition, the coupled quantities can in principle have non-reciprocal effects. ${ }^{17}$ For example, an electric current along the ferro-toroidal moment direction in materials with ferro-toroidal order can exhibit a nonreciprocal effect.

Note that the order parameter characterizing ferroelastic materials, i.e., strain, is a polar rank-2 tensor and thus does not belong to the vector order parameters described in Fig. 1.4,7,18 Here, neither space inversion nor time reversal symmetry is broken, but the rotational symmetry is broken. For instance, in a cubic to tetragonal transition the three-fold rotational symmetry is lost. ${ }^{4}$ Polar tensors relate vectors of the same type. More generally, tensors resulting from the product of an odd number of axial vectors with any number of polar vectors are called axial tensors. Optical gyration in certain crystals is an example of an axial tensor.
One can generalize the vector-table in Fig. 1 to rank-2 (and higher rank) tensors.

Chirality is the property of any three-dimensional object that cannot be superimposed with its mirror image, so handedness can be defined for a chiral object. Chirality is ubiquitous in nature, and it underpins many physical phenomena, such as optical activity. For the sake of simplicity, we will use "chirality" for proper screwtype "crystallographic or structural" distortions in this perspective. Crystals with chiral structure often consist of both left-handed and right-handed domains. For example, quartz forms in a chiral structure and the so-called Brazil twins consist of left-handed and right-handed domains. ${ }^{19}$ In chiral $\mathrm{Ni}_{3} \mathrm{TeO}_{6}$, three left-handed and three right-handed domains merge at one point, leading to an intriguing radiation-warning-sign pattern in a hexagonal-shape crystal. ${ }^{20}$ Chirality changes its handedness under space inversion, but remains intact under time reversal, similar to what happens with polarization in terms of invariance. However, for example, under mirror operations, chirality and polarization can behave differently.

Another interesting observation is the following: Under space inversion, "helical (proper screw type) spins" and "cycloidal spins" change their handedness or sense of rotation, but they do not change handedness or sense of rotation under time reversal. In fact, it turns out that under all five-type symmetry operations, "helical spins" and "cycloidal spins" can behave similarly like chirality and polarization in terms of invariance (whether it changes or is invariant), respectively. This is why cycloidal spins, but not helical spins alone, can lead to polarization in magnetismdriven ferroelectrics. In addition, as we will discuss below, "helical spins, combined with $\boldsymbol{M}$ (or $\boldsymbol{B}$ ) [i.e., helical spins $\otimes \boldsymbol{M}$ (or $\boldsymbol{B})$ ]" and "conical spins" can behave similar to "chirality $\otimes \boldsymbol{M}$ (or $\boldsymbol{B})$ " in terms of non-reciprocity.

Various shells exhibit ferro-rotational or chiral characteristics. Ancient mollusk shells such as nautilus (living fossil) and ammonites have planar spiral (rotational) shells (Fig. 2a), but most of the modern snail shells do exhibit either left or right chirality (Fig. 2b), which probably stems from the evolutionary stability of chiral structure. Interestingly, snail shells with right chirality (righthanded shells) turn out to be much more common (about 90\%) than those with left chirality. A large number of crystals do exhibit ferro-rotational characteristics, and often transform from a symmetric phase to a ferro-rotational phase. ${ }^{21-25}$ For example, the room-temperature structure (space group $\mathrm{P} \overline{3} \mathrm{~m} 1$ ) of $\mathrm{RbFe}$ $\left(\mathrm{MoO}_{4}\right)_{2}$ is built of $\mathrm{FeO}_{6}$ octahedra sharing vertices with $\mathrm{MoO}_{4}$ tetrahedra, which form layers arranged perpendicular to the $c$ axis with $\mathrm{Rb}$ atoms occupying the interlayer space, and it undergoes a phase transition with polyhedral rotations below $190 \mathrm{~K}$ (to space group P 3 ) with clockwise (left panel) or counterclockwise (right panel) ferro-rotational order (leading to ferro-rotational domains, Fig. 2b). ${ }^{22,23,26-28}$ It has been theoretically predicted that nanostructures of ferroelectrics can exhibit ferro-rotations of local electric dipoles. ${ }^{14}$ Indeed, the so-called polar vortices have been reported in a nanoscale-layered $\mathrm{PbTiO}_{3} / \mathrm{SrTiO}_{3}$ superstructure, ${ }^{29}$ even though their real structure is likely a chiral structure, rather than a ferro-rotational structure (Fig. 2c). Numerous patterns that we face every day do exhibit (ferro-)rotational characteristics. For example, the yin-yang symbol can have either a clockwise or a counterclockwise rotational pattern (Fig. 2d). We call the right(left-) panel pattern in Fig. 2d as yin (yang): yin (yang) is a mirror image of yang (yin) for a mirror perpendicular to the (ferro-) rotational plane, and yin and yang can be mutually converted to each other by a spatial rotation (i.e., yin and yang are topologically identical). Recall that neither space inversion nor time reversal is broken by (ferro-)rotation.

$\mathrm{RbFe}\left(\mathrm{MoO}_{4}\right)_{2}$ with $\mathrm{S}=5 / 2 \mathrm{Fe}^{3+}$ ions is a quasi-2D triangular antiferromagnet with weak XY anisotropy in the $a b$ plane. ${ }^{27}$ The $120^{\circ}$ spin order occurs in the $a b$ plane below $3.8 \mathrm{~K}$, and rotates in a helical manner among neighboring layers with an 

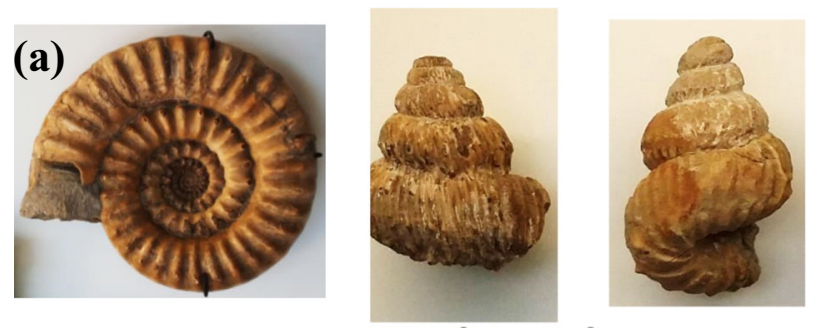

(b)
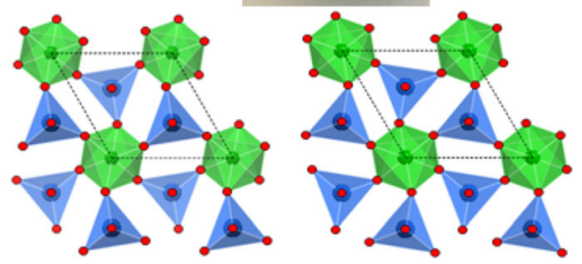

(c)

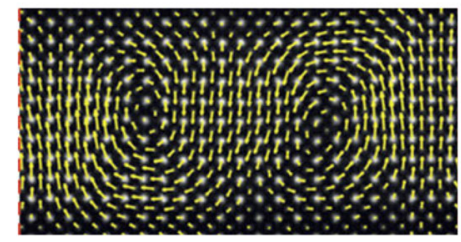

(d)

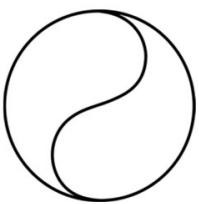

Yin

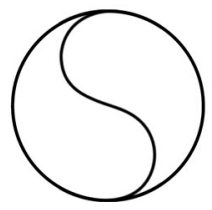

Yang
Fig. 2 a Shells with rotational (left panel), left chiral (middle panel), and right chiral (right panel) order (photos taken at the Oxford University Museum of Natural History). b Ferro-polyhedral rotations in the $\mathrm{RbFe}\left(\mathrm{MoO}_{4}\right)_{2}$ structure viewed along the $\boldsymbol{c}$ axis: no polyhedral rotation at room temperature, and polyhedral rotation in two types of ferro-rotational domains below $190 \mathrm{~K}$ (space group $\mathrm{P}$ 3) with clockwise (left panel) or counterclockwise (right panel) ferrorotational order. c Clockwise (left panel) and counterclockwise (right panel) ferro-rotations of local electric dipoles in a $\mathrm{PbTiO}_{3} / \mathrm{SrTiO}_{3}$ superstructure. d Clockwise (Yin, left panel) and counterclockwise (Yang, right panel) ferro-rotations in the yin-yang symbol). Emphasize that neither space inversion nor time reversal is broken by ferrorotation

incommensurate propagation vector $\mathrm{q}_{\mathrm{z}} \approx 0.44 .{ }^{28}$ It turns out that "ferro-rotation $\otimes$ helical spins" with a common axis do behave similar to polarization along the axis direction under all five symmetry operations in terms of invariance. Indeed, the appearance of polarization along the $c$ axis below $3.8 \mathrm{~K}$ has been experimentally observed. This again confirms the beauty as well as the power of symmetry considerations.

An object moving (with linear momentum or wave vector of $\boldsymbol{k}$ ) along one direction is reciprocal if the object moving along the opposite direction can be obtained through one or more consecutive operation(s) of the five symmetries (translational, rotational, mirror reflection, space inversion and time reversal). This object can be a particle or a quasi-particle in crystalline solids, including an electron, a hole, a phonon, a magnon or light. Note that we discuss first the objects with only linear momentum or wavevector without angular momentum (for example unpolarized light). First, we consider a ferromagnet: space inversion connects reciprocal situations for an object moving along magnetization (or the applied magnetic field) direction, so this case is reciprocal (Fig. 3a). For a chiral material, a simple spatial rotation (or time reversal) connects reciprocal situations for an object moving along the chiral crystallographic axis, so this case is also reciprocal (Fig. 3b). Note that for example, a right screw (a (a)
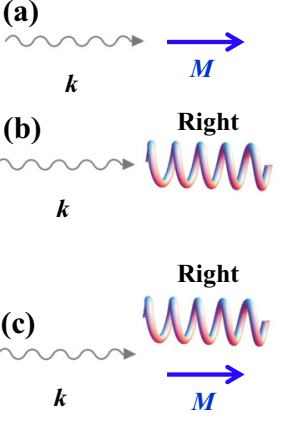

(d)

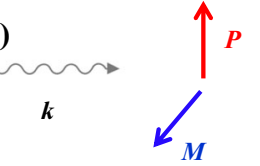

Space
Inversion

Time
Reversal$$
\text { sym }
$$
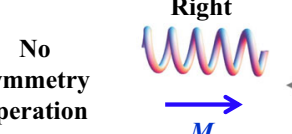

M

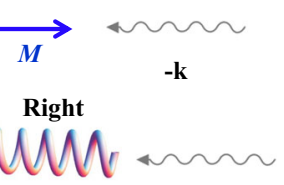

$-\mathbf{k}$

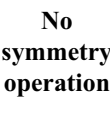

Fig. 3 Reciprocity and symmetry operations. a Space inversion links reciprocal situations for an object moving along magnetization or the applied magnetic field direction, so this case is reciprocal. b A simple spatial rotation (or time reversal) connects reciprocal situations for an object moving along the chiral axis, so this case is also reciprocal. c However, an object moving along the chiral axis is non-reciprocal if the chiral system is ferromagnetic with magnetization $(\boldsymbol{M})$ along the chiral axis or when a magnetic field is applied along the chiral axis, since two relevant reciprocal situations cannot be related by any one or combination of symmetry operations. d Similarly, an object moving along the ferro-toroidal vector direction $(\boldsymbol{P} \times \boldsymbol{M}$ direction) is non-reciprocal, since two relevant reciprocal situations cannot be linked by symmetry operations

chiral object) always remains to be a right screw with any spatial rotation. However, an object moving along the chiral axis is nonreciprocal if the chiral material is ferromagnetic with magnetization $(\boldsymbol{M})$ along the chiral axis or when a magnetic field $(\boldsymbol{B})$ is applied along the chiral axis, since two relevant reciprocal situations cannot be linked by any one or combination of five symmetry operations (Fig. 3c). On the other hand, chirality, combined with $\boldsymbol{P}$ (or $\boldsymbol{E}$ ) along the chiral axis, is still reciprocal, since time reversal still connects two reciprocal situations. Note that chirality with magnetization along the chiral axis or when a magnetic field is applied along the chiral axis, i.e., "chirality $\otimes \boldsymbol{M}$ (or $\boldsymbol{B}$ )", behaves similar to a velocity (or linear momentum, wavevector) along the chiral axis under space inversion and time reversal (in fact, under all five symmetry operations) in terms of invariance. Similarly, an object moving along the ferro-toroidal vector direction (the $\boldsymbol{P} \times \boldsymbol{M}$ direction) is non-reciprocal, since two relevant reciprocal situations cannot be connected by any one or combination of symmetry operations (Fig. 3d). On the other hand, all cases of $\boldsymbol{k}\|\boldsymbol{P}\| \boldsymbol{M}, \boldsymbol{k} \perp \boldsymbol{P}\|\boldsymbol{M}, \boldsymbol{k}\| \boldsymbol{P} \perp \boldsymbol{M}$, and $\boldsymbol{k} \| \boldsymbol{M} \perp \boldsymbol{P}$ are reciprocal, since two relevant reciprocal situations can be linked by symmetry operations (see Supplementary Information, Fig. S1).

Carbon nanotubes are chiral molecular objects (chiral axis matches with the tube axis), and the charge transport through individual single-walled carbon nanotubes in the presence of a magnetic field parallel to the tube axis turns out to be directiondependent: resistance along one tube-axis direction changes by about $0.3 \%$ at $1.5 \mathrm{~K}$ when the orientation of magnetic field of 14 tesla along the tube axis is switched by $180^{\circ} .{ }^{30}$ This directiondependent magnetoresistance is called electrical magneto-chiral anisotropy, and is consistent with our discussion of Fig. 3c. Note that quasi-equilibrium states with drift velocities are relevant to these magneto-chiral anisotropy effects, $p-n$ junction diode effects, as well as ferroelectric diode effects (see Supplementary 
Information, Fig. S2). In this perspective, we are mainly concerned with (quasi-)equilibrium phenomena. We defer the discussion of non-equilibrium phenomena and related Onsager reciprocity relations to future investigations.

In magnetic materials, elementary excitations are magnons (spin waves). These quasiparticles are actively studied as spin current carriers for potential applications in spintronics, where the spin current serves as a Joule-heating-free alternative to an electric current. Nonreciprocal magnonic effects could potentially be used for creating new types of spintronic devices, such as spin current diodes. The chiral ferromagnet concept shown in Fig. $3 \mathrm{c}$ provides an important example of a system with non-reciprocal magnons. The chirality in this figure can be a pure crystallographic chirality or due to helical spins. As discussed earlier, structural chirality and helical spins act similarly under all five-symmetry operations in terms of invariance. A classic example of such a purely magnetic system (helical spins $\otimes \boldsymbol{M}$ ) is the conical state in metal erbium, in which the spins rotate around the net magnetic moment pointing along the $\mathrm{c}$ axis. There, the dispersion curves for the magnons propagating in the $+\mathrm{c}$ and $-\mathrm{c}$ directions show significant differences over a wide wavevector range. ${ }^{31}$ For spintronic applications, however, collinear ferromagnetic insulators are more relevant. Recent observation of nonreciprocal magnons in $\mathrm{Cu}_{2} \mathrm{OSeO}_{3}$ provides a good example of such a system. $\mathrm{Cu}_{2} \mathrm{OSeO}_{3}$ exhibits a chiral crystal structure, as well as a collinear ferromagnetic state in a weak applied magnetic field. Nonreciprocal effects are observed ${ }^{32}$ when the magnetization is aligned with the chiral crystallographic axis, in full agreement with the scenario shown in Fig. 3c. This example points to an important advantage of the magnonic effects. Magnetic field is a strong control tool for these effects because it couples to the magnons directly. In the chiral ferromagnet example, the non-reciprocal effects can be switched on and off by rotating the magnetization direction, which can be achieved readily. More complex magnetic systems provide an even more fertile ground for non-trivial magnon/light propagation effects. Recent examples include magneto-chiral dichroism in $\mathrm{Ba}_{2} \mathrm{CoGe}_{2} \mathrm{O}_{7},{ }^{33}$ as well as the opposite spontaneous magnonic Faraday rotation of the counterpropagating spin waves in $\mathrm{a}-\mathrm{Cu}_{2} \mathrm{~V}_{2} \mathrm{O}_{7}{ }^{34}$

For observations of non-reciprocal effects in optics, one introduces the concept of non-reciprocal directional anisotropy that distinguishes between the two opposite propagation directions and does not depend on the polarization of light (in general, spin or orbital angular momentum). ${ }^{1,6}$ Thus, the nonreciprocal directional anisotropy is more narrowly defined than general non-reciprocity, e.g., it excludes the Faraday effect (see Supplementary Information). The experimental manifestations of the non-reciprocal directional anisotropy are the non-reciprocal directional dichroism and the non-reciprocal directional birefringence, which are related by the Kramers-Kronig transformation. The non-reciprocal directional dichroism refers to the difference in the absorption coefficient for counter-propagating light beams, while the non-reciprocal directional birefringence denotes the difference in the real part of the refractive index in the same situation. To observe the non-reciprocal directional anisotropy experimentally, there must exist a symmetry operator that is responsible for changing the sign of one relevant property tensor, resulting in the change of the phase of induced polarization $P(\omega, k)$ and magnetization $M(\omega, k)$, while the other property tensor remains unchanged. An intuitive example is the case of a chiral ferromagnet, where both optical activity and Faraday effect may manifest. Each effect can be described by its own gyration vector (denoted here as $\vec{G}$ for optical activity and $\vec{M}$ for Faraday effect) and written in the form $\vec{D} \propto i \varepsilon_{0}(\vec{G}+\vec{M}) \times \vec{E}$, where the cross product on the right can always be represented by the product of an antisymmetric tensor $[G+M]$ with $\vec{E}$. Of the two parts of the tensor, Mchanges sign and $G$ does not when the magnetization is reversed, leading to polarization-independent directional anisotropy.

In practice, the non-reciprocal directional birefringence results in a different phase of light propagating in the opposite direction, which is not easily observed. Thus, the directional anisotropy is most readily detected via the non-reciprocal directional dichroism in absorbing media. ${ }^{1}$ We use the term optical diode effect (ODE) to denote the directional dichroism. Let the matrix $t_{i j}$ connect the incident and transmitted electric field of the light wave via $E_{x}^{t}=$ $t_{x x} E_{x}^{i}+t_{x y} E_{y}^{i}$ and $E_{y}^{t}=t_{y x} E_{x}^{i}+t_{y y} E_{y}^{i}$, where $E_{x}^{i}$ and $E_{y}^{i}$ are the orthogonal components of the incident photon field. The ODE can be measured as the ratio of transmitted intensities $\left|t_{x x}(+k)\right|^{2} /\left|t_{x x}(-k)\right|^{2}$ (or $\left|t_{y y}(+k)\right|^{2} /\left|t_{y y}(-k)\right|^{2}$ ) along the $+\boldsymbol{k}$ and $\boldsymbol{- k}$ directions. This definition excludes the non-reciprocity due to the Faraday effect and obeys all of the symmetry considerations discussed in this perspective and illustrated in Fig. 3.

As an example, we consider the $\mathrm{THz}$ ODE in polar ferromagnet ${ }^{35-37} \mathrm{FeZnMo}_{3} \mathrm{O}_{8}$, where it results from the presence of the toroidal vector $\boldsymbol{T}=\boldsymbol{P} \times \boldsymbol{M}$ when light propagates along $\boldsymbol{T}(\boldsymbol{k} \| \boldsymbol{T}$, Fig. 5). Crystallographic $\boldsymbol{c}$ axis represents the polar direction $\boldsymbol{P}$. The toroidal vector develops as magnetic field $\boldsymbol{B}$ is applied perpendicular to the $\boldsymbol{c}$ axis [see Supplementary information for details]. Figure $5 \mathrm{a}$ displays the observation of the giant $\mathrm{THz}$ ODE in $\mathrm{FeZnMO}_{3} \mathrm{O}_{8}$ measured as the ratio of transmitted intensities for positive and negative applied magnetic field $\boldsymbol{B}$, which is identical to the measurement with positive and negative $\boldsymbol{k}$ from the broken symmetry standpoint. The incident light is linearly polarized with electric field $E^{i}$ along the $c$ axis. In zero field, the resonance at 1.27 THz corresponds to a spin excitation across the anisotropy gap, ${ }^{37}$ Fig. 5a. Remarkably, as magnetic field is applied perpendicular to the $\boldsymbol{c}$ axis, this spin resonance loses strength and disappears for negative $\boldsymbol{B}$, while it gains significant strength for positive $\boldsymbol{B}$. The crystal becomes completely transparent for light traveling in one direction and completely opaque in the opposite propagation direction, with the ratio $\left|t_{x x}(+k)\right|^{2} /\left|t_{x x}(-k)\right|^{2}$ in excess of 100. We find significant ODE at $110 \mathrm{~K}$, a much higher temperature than the Curie temperature of the material $T_{C}=$ $14 \mathrm{~K}$, Fig. 5b. All prior observations of THz ODE in magnetoelectric multiferroics were in a magnetic state with long-range order, often at very low temperatures. ${ }^{38-41}$ In $\mathrm{FeZnMo}_{3} \mathrm{O}_{8}$, the ODE remains strong in the high temperature paramagnetic state without magnetic order because it occurs at the frequency of the anisotropy gap excitation governed by the single-ion magnetic and magnetoelectric Hamiltonian. These interactions open up a promising avenue in the search for high-temperature (especially room-temperature) ODE in other paramagnetic crystals. ${ }^{37}$

A large number of compounds form in the non-polar but ferrorotational crystallographic structure whose point symmetry can be one of $\overline{7}, 2 / m, \overline{3}, \overline{4}, \overline{6}, 4 / m$, and $6 / \mathrm{m}^{24}$ Examples include $\mathrm{NaBi}$ $\left(\mathrm{MoO}_{4}\right)_{2}\left(14_{1} / a\right.$, belonging to $4 / \mathrm{m}$, above $\left.241 \mathrm{~K}\right){ }^{42} \mathrm{PbMoO} 4\left(14_{1} /\right.$ $a),{ }^{43} \mathrm{Cu}_{3} \mathrm{Nb}_{2} \mathrm{O}_{8}(\mathrm{P} \overline{7}),{ }^{24} \mathrm{CaMn}_{7} \mathrm{O}_{12}(R \overline{3}$ below $440 \mathrm{~K}),{ }^{25}$ and RbFe $\left(\mathrm{MoO}_{4}\right)_{2}\left(P \frac{2}{3}\right.$ below $\left.190 \mathrm{~K}\right) .^{22,26}$ As we discussed earlier, $\mathrm{d} B / \mathrm{d} t$ or the monopole current can be a conjugate field for the ferrorotational order, but appropriate experimental realizations of the coupling effects are not evident. For example, many of the ferrorotational compounds undergo a phase transition from a symmetric phase to a ferro-rotational phase, so clockwise and counterclockwise ferro-rotational domains should exist, but have not been observed yet, as far as we know.

Various cases for (non-)reciprocity in ferro-rotational order are depicted in Fig. 4. Time reversal connects reciprocal situations for an object moving along the ferro-rotational axis, so this case is reciprocal (Fig. 4a). Adding polarization or an electric field along the ferro-rotational axis does not change the situation. When we add magnetization or a magnetic field along the ferro-rotational axis, the connecting symmetry simply changes from time reversal to a mirror reflection, so this case is still reciprocal. However, ferrorotational order with simultaneous presence of $\boldsymbol{P}$ (or $\boldsymbol{E}$ ) and $\boldsymbol{M}$ (or 
(a)<smiles>C1C2CC3CC1CC(C2)C3</smiles><smiles>C1C2CC3CC1CC3C2</smiles>
$\sim \sim$ $\boldsymbol{k}$ Time reversal

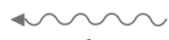

$-k$

(b)

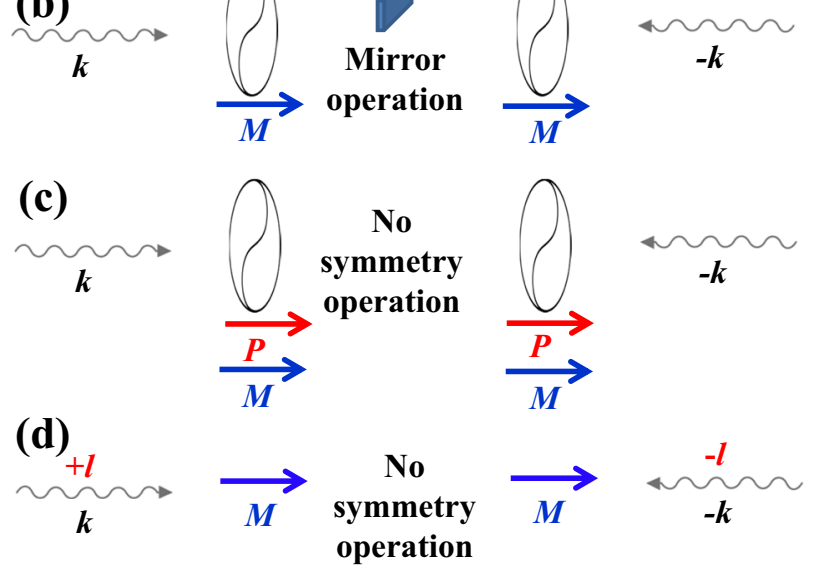

Fig. 4 Reciprocity of ferro-rotational ("Yin"-type) materials, including the propagation of objects with angular momentum. See the main text for the definition of "Yin" and "Yang" in this perspective. a Time reversal connects reciprocal situations for an object moving perpendicular to the ferro-rotational plane, so this case is reciprocal. b Even if the ferro-rotational system is ferromagnetic with magnetization $(\boldsymbol{M})$ along the ferro-rotational axis, it is reciprocal since two reciprocal situations can be connected by a mirror operation. In addition, ferro-rotation, combined with polarization along the ferro-rotational axis, is reciprocal, since time reversal links two relevant reciprocal situations. c However, ferro-rotation, combined with magnetization $(\boldsymbol{M})$ and polarization along the ferro-rotational axis, can be non-reciprocal since two reciprocal situations cannot be related by any symmetry operation. d An object with non-zero angular momentum moving along the magnetization $(\boldsymbol{M})$ direction of a ferro(ferri)magnet can be nonreciprocal, since two relevant reciprocal situations cannot be connected by symmetry operations

B) along the ferro-rotational axis can now exhibit a non-reciprocal effect, since no symmetry links the two reciprocal situations. In fact, the simultaneous presence of ferro-rotation and polarization along the ferro-rotation axis, behaves similar to chirality under all of the five symmetry operations. Thus, ferro-rotation, combined with $\boldsymbol{P}$ (or $\boldsymbol{E}$ ) and also $\boldsymbol{M}$ (or $\boldsymbol{B}$ ) along the ferro-rotational axis, just like chirality combined with $\boldsymbol{M}$ (or $\boldsymbol{B}$ ) along the chirality axis, can show non-reciprocity. Therefore, a non-reciprocal effect can exist in a single domain of ferro-rotational order in the simultaneous presence of $\boldsymbol{P}$ (or $\boldsymbol{E}$ ) and $\boldsymbol{M}$ (or $\boldsymbol{B}$ ) along the ferro-rotational axis, and ferro-rotational domains can be detected in the simultaneous presence of $\boldsymbol{P}$ (or $\boldsymbol{E}$ ) and $\boldsymbol{M}$ (or $\boldsymbol{B}$ ). Note that under space inversion and time reversal operations, all non-reciprocal cases of Figs. $3 c$, d and $4 \mathrm{c}$ act like velocity (or linear momentum, wavevector), i.e., it changes their character under either space inversion or time reversal, which is the origin of non-reciprocity in all three cases (in fact, this argument works for all symmetry operations). Note also that chirality induces optical activity, i.e., the rotation of the polarization direction of linearly polarized light when the light travels through a chiral material. Similarly, ferro-rotation with $\boldsymbol{P}$ (or $\boldsymbol{E})$ can induce optical activity. In fact, it is known that the optical activity of a ferro-rotational material such as $\mathrm{NaBi}\left(\mathrm{MoO}_{4}\right)_{2}$ and $\mathrm{PbMoO}_{4}$ increases linearly with an applied electric field, which is known as linear gyration. In principle, this linear gyration can be utilized to observe ferro-rotational domains.

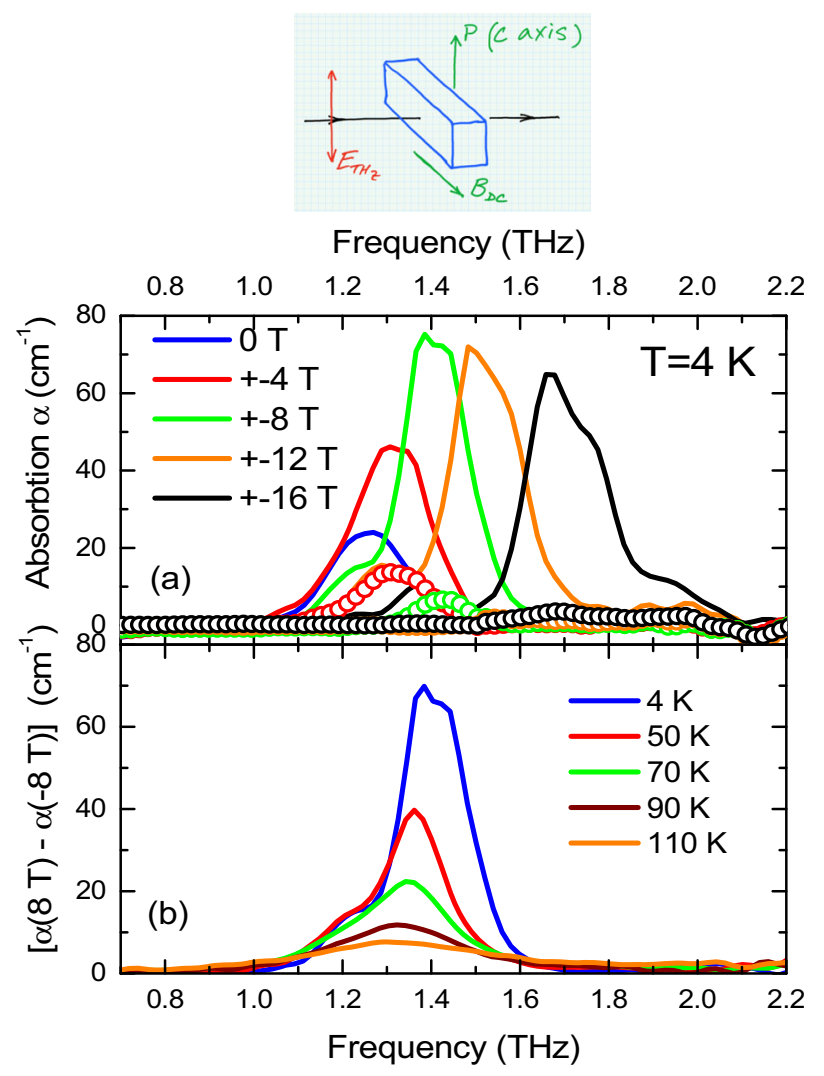

Fig. 5 Optical diode effect in the polar ferromagnet $\mathrm{FeZnMo}_{3} \mathrm{O}_{8}$. The ferromagnetic ordering temperature $T_{C}=14 \mathrm{~K}$. Optical diode effect was observed in the geometry shown in the top panel (corresponding to Fig. $3 \mathrm{~d}$ ), where the light propagates along and opposite to the toroidal vector $\boldsymbol{P} \times \boldsymbol{M}$. The polarization vector $\boldsymbol{P}$ is along the crystallographic $\boldsymbol{c}$ axis. The magnetic direction $\boldsymbol{M}$ is set by applying an external magnetic field perpendicular to $\boldsymbol{P}$. a Magnetic field dependence of the optical diode effect. No effect is observed in zero field. The absorption resonance at $1.27 \mathrm{THz}$ in zero field is due to a spin excitation across the anisotropy gap. The spin resonance frequency increases with the magnetic field. At the same time, the resonant absorption is almost entirely suppressed for one direction of the magnetic field and is greatly enhanced for the opposite magnetic field direction. By symmetry, this is equivalent to nonreciprocal light propagation. Solid lines show the positive magnetic field, open symbols show the negative magnetic field. b Temperature dependence of the optical diode effect, which remains strong even far above $T_{c}$. Figures are adapted with permission from ref. 37, copyright 2018 American Physical Society

So far, we have discussed mostly the motion of objects without angular momentum, such as un-polarized or linearly polarized light. The symmetry approach works, of course, when angular momentum or the circular polarization is added. This significantly increases the range of the possible nonreciprocal effects. Circularly-polarized light possesses spin angular momentum. In addition, a vortex beam of light or electrons is twisted like a screw around its axis of travel, and has orbital angular momentum. An object with angular momentum propagating, for example, along the ferro-rotational (or chiral) axis is reciprocal, since two reciprocal situations can be related through time reversal. For this reason, the optical activity of chiral materials is reciprocal, and the polarization rotation of linearly polarized light is the same for two opposite propagation directions along the chiral axis. However, when time reversal symmetry is broken in a material, the situation changes; for example, an object with angular momentum propagating along the magnetization direction is non-reciprocal, since two reciprocal situations cannot be related 
(a)

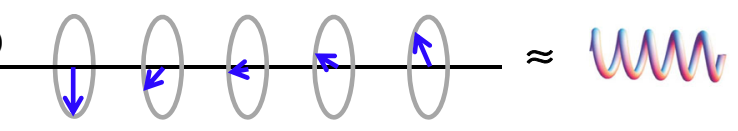

(b)

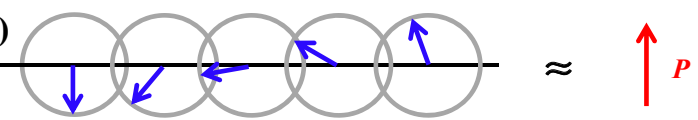

(c)

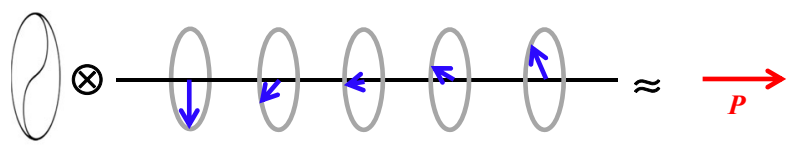

(d)

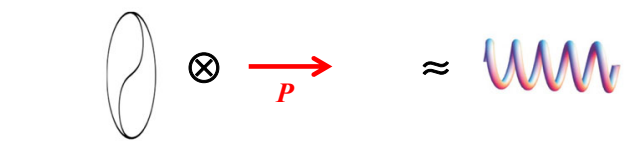

(e)

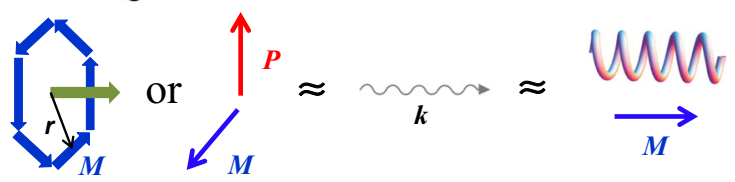

Fig. 6 Different entities behave similarly under all five symmetry operations (translation, rotation, mirror reflection, space inversion and time reversal) in terms of invariance (whether it changes or is invariant). a The handedness of helical spins behaves identically with that of chirality with chiral axis along the helical axis. b Cycloidal spins act similar to polarization in the cycloidal plane and perpendicular to the cycloidal modulation direction. c Ferrorotation, combined with helical spins with helical axis along the ferro-rotational axis, behaves similar to polarization along the ferrorotational axis. d Ferro-rotation, combined with polarization along the ferro-rotational axis, acts similar to chirality with chiral axis along the ferro-rotational axis. e Ferro-toroidal moment behaves similar to velocity (linear momentum, wavevector) under all symmetry operations, which is the origin of common non-reciprocity in ferro-toroidal materials

through any symmetry operation(s) (Fig. 4d). In fact, this is the origin of the Faraday rotation (see Supplementary Information). As far as we know, the Faraday rotation of a vortex beam with orbital angular momentum in ferromagnetic (or ferrimagnetic) materials has not been investigated, and can be an interesting topic for future study.

Figure 6 summarizes five entities that behave similarly under all five symmetry operations in terms of invariance (whether it changes or is invariant), discussed in this perspective. The handedness of helical spins behaves identically with that of chirality under all five symmetry operations, while cycloidal spins and polarization in the cycloidal plane and perpendicular to the cycloidal modulation direction act similarly under all symmetry operations in terms of invariance (Fig. 6a, b). This is why the cycloidal spin order can result in polarization (through the microscopic mechanism of inverse Dzyaloshinskii-Moriya interaction), but the helical spin order itself does not. Ferro-rotation, combined with helical spins with helical axis coinciding with the ferro-rotational axis, behaves similar to polarization along the ferro-rotational axis under all symmetry operations, which leads to the so-called ferro-axial multiferroicity (Fig. 6c). ${ }^{44}$ Ferro-rotation, combined with polarization along the ferro-rotational axis, behaves like chirality under all five symmetry operations. This is the origin of why both "ferro-rotation, combined with $\boldsymbol{P}$ (or $\boldsymbol{E}$ ) along the ferro-rotational axis and $\boldsymbol{M}$ (or $\boldsymbol{B}$ ) along the ferrorotational axis" and "chirality, combined with $\boldsymbol{M}$ (or $\boldsymbol{B}$ ) along the chiral axis" exhibit non-reciprocal effects for an object moving along the ferro-rotational or chiral axis. Evidently, "conical spins", which is like "helical spins with $\boldsymbol{M}$ (or $\boldsymbol{B}$ ) along the helical axis", and "chirality, combined with $\boldsymbol{M}$ (or $\boldsymbol{B}$ ) along the chiral axis", can exhibit a non-reciprocal effect. Finally we emphasize that ferrotoroidal moment behaves similar to velocity (linear momentum, wavevector) under all symmetry operations, which is the origin of common non-reciprocity in ferro-toroidal materials. Therefore, these similarities under symmetry operations in Fig. 6 are relevant to numerous non-trivial physical phenomena that we have discussed. In addition, the non-reciprocity in ferro-toroidal materials implies that ferro-toroidal domains can be observable in transmission polarized optical microscopy, and the similarity between helical spins and chirality suggests that the magnetic domains associated with helical spins can be observed under a transmission polarized optical microscope, even though the magnitude of the effects cannot be predicted from symmetry arguments.

In conclusion, we have demonstrated through a series of illustrative examples how non-reciprocal behavior can be realized in ferroic and multiferroic materials with a vector order parameter, in particular ferro-rotational and ferro-toroidal orders. In short, if a situation with a velocity and its reciprocal situation cannot be linked through any symmetry operations, then the situation can be non-reciprocal. Alternatively, we can state that if a material has symmetry operation properties identical with those of a velocity in terms of invariance, then a motion of an object with the velocity in the material can be non-reciprocal. These findings have direct implications for various attendant topological defects, specifically the detection of ferro-rotational and other domain walls. The concepts of using linear optical gyration (and possibly vortex beams) for domain wall detection, optical diode effect (e.g., in polar ferrimagnet $\mathrm{FeZnMo}_{3} \mathrm{O}_{8}$ ) and how to achieve it at high temperature, as well as the interplay of ferro-rotation, chirality and helical/cycloidal spin configurations in the context of other nonreciprocal effects were explored. How to utilize vortex beams of electrons or light to explore the coupling between orbital angular momentum and materials with various broken symmetries needs to be further explored. How to utilize vortex beams of electrons or light to explore the coupling between orbital angular momentum and materials with various broken symmetries needs to be further explored. We also note that our consideration of symmetries and reciprocity can be further expanded to higher-order-rank tensorial orders such as strain and also staggered quantities such as antiferro-rotational and antiferro-toroidal orders. These notions will allow the design of new materials with desired multiferroic, optical and non-reciprocal properties and delineate how to probe their unusual domain walls.

\section{ACKNOWLEDGEMENTS}

S.W.C. and V.K. are supported by the DOE under Grant No. DOE: DE-FG02-07ER46382. D.T. is supported by the NSF Award No. DMR-1554866. The work of A.S. is supported by the US DOE. We have greatly benefited by discussions with Fei-Ting Huang, SangHyuk Lee, Andrei Sirenko, Oleg Tchernyshyov, Maxim Mostovoy, Daniel Khomskii, and David Vanderbilt.

\section{AUTHOR CONTRIBUTIONS}

S.W.C. advised the conception of this article and oversaw the overall structure of the manuscript. All authors contribute to the writing and revision of the manuscript.

\section{ADDITIONAL INFORMATION}

Supplementary information accompanies the paper on the npj Quantum Materials website (https://doi.org/10.1038/s41535-018-0092-5).

Competing interests: The authors declare no competing interests.

Publisher's note: Springer Nature remains neutral with regard to jurisdictional claims in published maps and institutional affiliations. 


\section{REFERENCES}

1. Rikken, G. L. J. A., Strohm, C. \& Wyder, P. Observation of magnetoelectric direc tional anisotropy. Phys. Rev. Lett. 89, 133005 (2002).

2. Choi, T., Lee, S., Choi, Y. J., Kiryukhin, V. \& Cheong, S.-W. Switchable ferroelectric diode and photovoltaic effect in $\mathrm{BiFeO}_{3}$. Science 324, 63-66 (2009).

3. Cheong, S.-W. \& Mostovoy, M. Multiferroics: a magnetic twist for ferroelectricity. Nat. Mater. 6, 13-20 (2007).

4. Saxena, A. Broken symmetry, ferroic phase transitions and multifunctional materials. Integr. Ferroelectr. 131, 3-24 (2011).

5. Hlinka, J. Eight types of symmetrically distinct vectorlike physical quantities. Phys. Rev. Lett. 113, 165502 (2014).

6. Szaller, D., Bordács, S. \& Kézsmárki, I. Symmetry conditions for nonreciprocal light propagation in magnetic crystals. Phys. Rev. B 87, 014421 (2013).

7. Saxena, A. \& Lookman, T. Magnetic symmetry of low-dimensional multiferroics and ferroelastics. Phase Trans. 84, 421-437 (2011).

8. Schmid, H. On ferrotoroidics and electrotoroidic, magnetotoroidic and piezotoroidic effects. Ferroelectrics 252, 41-50 (2001).

9. Spaldin, N. A., Fiebig, M. \& Mostovoy, M. The toroidal moment in condensedmatter physics and its relation to the magnetoelectric effect. J. Phys. Condens. Matter 20, 434203 (2008)

10. Dubrovik, V. M. \& Tugushev, V. V. Toroid moments in electrodynamics and solidstate physics. Phys. Rep. 187, 145-202 (1990).

11. Kopaev, Yu. V. Toroidal ordering in crystals. Phys. Usp. 52, 1111-1125 (2009).

12. Ederer, K. \& Spaldin, N. A. Towards a microscopic theory of toroidal moments in bulk periodic crystals. Phys. Rev. B 76, 214404 (2007).

13. Muthukumar, V. N., Valentí, R. \& Gros, C. Theory of nonreciprocal optical effects in antiferromagnets: The case of $\mathrm{Cr}_{2} \mathrm{O}_{3}$. Phys. Rev. B 54, 433 (1996).

14. Prosandeev, S., Ponomareva, I., Naumov, I., Kornev, I. \& Bellaiche, L. Original properties of dipole vortices in zero-dimensional ferroelectrics. J. Phys. Condens. Matter 20, 193201 (2008)

15. Aken, B. B. Van, Rivera, J. P., Schmid, H. \& Fiebrig, M. Observation of ferrotoroidic domains. Nature 449, 702-705 (2007).

16. Catalan, G., Seidel, J., Ramesh, R. \& Scott, J. F. Domain wall nanoelectronics. Rev. Mod. Phys. 84, 119-156 (2012).

17. Miyahara, S. \& Furukawa, N. Nonreciprocal directional dichroism and toroidalmagnons in helical magnets. J. Phys. Soc. Jpn. 81, 023712 (2012).

18. Aizu, K. Possible species of "ferroelastic" crystals and of simultaneously ferroelectric and ferroelastic crystals. J. Phys. Soc. Jpn. 27, 387-396 (1969).

19. Sunagawa, I., Taijing, L. \& Balitsky, V. S. Generation of Brazil and Dauphiné twins in synthetic amethysts. Phys. Chem. Miner. 17, 320-325 (1990)

20. Wang, X. W., Huang, F.-T., Yang, J.-J., Oh, Y. S. \& Cheong, S.-W. Interlocked chiral/ polar domain walls and large optical rotation in $\mathrm{Ni}_{3} \mathrm{TeO}_{6}$. APL Mater. 3, 076105 (2015).

21. Gopalan, V. \& Litvin, D. B. Rotation-reversal symmetries in crystals and handed structures. Nat. Mater. 10, 376-381 (2011).

22. Kenzelmann, M. et al. Direct transition from a disordered to a multiferroic phase on a triangular lattice. Phys. Rev. Lett. 98, 267205 (2007).

23. Wáskowska, A. et al. Temperature- and pressure-dependent lattice behaviour of $\mathrm{RbFe}\left(\mathrm{MoO}_{4}\right)_{2}$. J. Phys. Condens. Matter 22, 055406 (2010).

24. Johnson, $\mathrm{R}$. D. et al. $\mathrm{Cu}_{3} \mathrm{Nb}_{2} \mathrm{O}_{8}$ : a multiferroic with chiral coupling to the crystal structure. Phys. Rev. Lett. 107, 137205 (2011).

25. Johnson, R. D. et al. Giant improper ferroelectricity in the ferroaxial magnet $\mathrm{CaMn}_{7} \mathrm{O}_{12}$. Phys. Rev. Lett. 108, 067201 (2012).

26. Inami, T. Neutron powder diffraction experiments on the layered triangularlattice antiferromagnets $\mathrm{RbFe}\left(\mathrm{MoO}_{4}\right)_{2}$ and $\mathrm{CsFe}\left(\mathrm{SO}_{4}\right)_{2}$. J. Solid State Chem. 180, 2075-2079 (2007).

27. White, J. S. et al. Multiferroicity in the generic easy-plane triangular lattice antiferromagnet RbFe( $\left(\mathrm{MoO}_{4}\right)_{2}$. Phys. Rev. B 88, 060409 (2013).
28. Hearmon, A. J. et al. Electric field control of the magnetic chiralities in ferroaxial multiferroic RbFe( $\left.\mathrm{MoO}_{4}\right)_{2}$. Phys. Rev. Lett. 108, 237201 (2012).

29. Yadav, A. K. et al. Observation of polar vortices in oxide superlattices. Nature $\mathbf{5 3 0}$, 198-201 (2016).

30. Krstić, V., Roth, S., Burghard, M., Kern, K. \& Rikken, G. L. J. A. Magneto-chiral anisotropy in charge transport through single-walled carbon nanotubes. J. Chem Phys. 117, 11315-11319 (2002).

31. Nicklow, R. M., Wakabayashi, N., Wilkinson, M. K. \& Reed, R. E. Spin-wave dispersion relation for Er metal at $4.5^{\circ} K^{*}$. Phys. Rev. Lett. 27, 334 (1971).

32. Seki, S. et al. Magnetochiral nonreciprocity of volume spin wave propagation in chiral-lattice ferromagnets. Phys. Rev. B 93, 235131 (2016).

33. Bordács, S. et al. Chirality of matter shows up via spin excitations. Nat. Phys. 8 , 734-738 (2012).

34. Gitgeatpong, G. et al. Nonreciprocal magnons and symmetry-breaking in the noncentrosymmetric antiferromagnet. Phys. Rev. Lett. 119, 047201 (2017).

35. Wang, Y. et al. Unveiling hidden ferrimagnetism and giant magnetoelectricity in polar magnet $\mathrm{Fe}_{2} \mathrm{Mo}_{3} \mathrm{O}_{8}$. Sci. Rep. 5, 12268 (2015).

36. Kurumaji, T., Ishiwata, S. \& Tokura, Y. Doping-tunable ferromagnetic phase with large linear magnetoelectric effect in a polar magnet $\mathrm{Fe}_{2} \mathrm{Mo}_{3} \mathrm{O}_{8}$. Phys. Rev. X 5, 031034 (2015)

37. $\mathrm{Yu}, \mathrm{S}$. et al. High-temperature terahertz optical diode effect without magnetic order in polar $\mathrm{FeZnMo}_{3} \mathrm{O}_{8}$. Phys. Rev. Lett. 120, 037601 (2018).

38. Miyahara, S. \& Furukawa, N. Theory of magnetoelectric resonance in twodimensional $\mathrm{S}=3 / 2$ antiferromagnet $\mathrm{Ba}_{2} \mathrm{CoGe}_{2} \mathrm{O}_{7}$ via spin-dependent metalligand hybridization mechanism. J. Phys. Soc. Jpn. 80, 073708 (2011).

39. Kézsmárki, I. et al. Enhanced directional dichroism of terahertz light in resonance with magnetic excitations of the multiferroic $\mathrm{Ba}_{2} \mathrm{CoGe}_{2} \mathrm{O}_{7}$ oxide compound. Phys. Rev. Lett. 106, 057403 (2011).

40. Kézsmárki, I. et al. Optical diode effect at spin-wave excitations of the roomtemperature multiferroic $\mathrm{BiFeO}_{3}$. Phys. Rev. Lett. 115, 127203 (2015).

41. Kuzmenko, A. M. et al. Large directional optical anisotropy in multiferroic ferroborate. Phys. Rev. B 92, 184409 (2015).

42. Wáskowska, A. et al. Low-temperature and high-pressure structural behaviour of $\mathrm{NaBi}\left(\mathrm{MoO}_{4}\right)_{2}$-an X-ray diffraction study. J. Solid State Chem. 178, 2218-2224 (2005).

43. Vesselinov, I. Relation between the structure of wulfenite, $\mathrm{PbMoO}_{4}$, as an example of scheelite type structure, and the morphology of its crystals. J. Cryst. Growth 10, 45-55 (1971).

44. Hlinka, J., Privratska, J., Ondrejkovic, P. \& Janovec, V. Symmetry guide to ferroaxial transitions. Phys. Rev. Lett. 116, 177602 (2016)

Open Access This article is licensed under a Creative Commons Attribution 4.0 International License, which permits use, sharing, adaptation, distribution and reproduction in any medium or format, as long as you give appropriate credit to the original author(s) and the source, provide a link to the Creative Commons license, and indicate if changes were made. The images or other third party material in this article are included in the article's Creative Commons license, unless indicated otherwise in a credit line to the material. If material is not included in the article's Creative Commons license and your intended use is not permitted by statutory regulation or exceeds the permitted use, you will need to obtain permission directly from the copyright holder. To view a copy of this license, visit http://creativecommons. org/licenses/by/4.0/.

(c) The Author(s) 2018 